\title{
The deep inspiration breath hold technique using Abches reduces cardiac dose in patients undergoing left-sided breast irradiation
}

\author{
Ha Yoon Lee, MD, Jee Suk Chang, MD, Ik Jae Lee, MD, PhD, Kwangwoo Park, PhD, Yong Bae Kim, MD, PhD, \\ Chang Ok Suh, MD, PhD, Jun Won Kim, MD, Ki Chang Keum, MD, PhD \\ Department of Radiation Oncology, Yonsei University College of Medicine, Seoul, Korea
}

Purpose: We explored whether the deep inspiration breath hold (DIBH) technique using Abches during left-sided breast irradiation was effective for minimizing the amount of radiation to the heart and lung compared to free breathing (FB).

Materials and Methods: Between February and July 2012, a total of 25 patients with left-sided breast cancer underwent two computed tomography scans each with the DIBH using Abches and using FB after breast-conserving surgery. The scans were retrospectively replanned using standardized criteria for the purpose of this study. The DIBH plans for each patient were compared with FB plans using dosimetric parameters.

Results: All patients were successfully treated with the DIBH technique using Abches. Significant differences were found between the DIBH and FB plans for mean heart dose (2.52 vs. $4.53 \mathrm{~Gy})$, heart V30 (16.48 vs. $\left.45.13 \mathrm{~cm}^{3}\right)$, V20 (21.35 vs. $\left.54.55 \mathrm{~cm}^{3}\right)$, mean left anterior descending coronary artery (LAD) dose (16.01 vs. $26.26 \mathrm{~Gy}$, all $p<0.001$ ), and maximal dose to $0.2 \mathrm{~cm}^{3}$ of the LAD (41.65 vs. $47.27 \mathrm{~Gy}, \mathrm{p}=0.017)$. The mean left lung dose (7.53 vs. $8.03 \mathrm{~Gy}, \mathrm{p}=0.073)$ and lung V20 (14.63\% vs. $15.72 \%, \mathrm{p}=0.060)$ of DIBH using Abches were not different significantly compared with FB.

Conclusion: We report that the use of a DIBH technique using Abches in breathing adapted radiotherapy for left-sided breast cancer is easily feasible in daily practice and significantly reduces the radiation doses to the heart and LAD, therefore potentially reducing cardiac risk.

Keywords: Abches, Deep inspiration breath hold, Cardiac toxicity

\section{Introduction}

Postoperative radiotherapy for breast cancer patients has been shown to significantly reduce the risk of local recurrence as well as improving long-term survival $[1,2]$. However, many studies have demonstrated increased cardiac mortality and morbidity after breast radiotherapy [3-5]. Although many of the studies included in the this review involved old treatment techniques, which probably delivered a higher dose to the heart than seen in modern radiotherapy clinics, the issue of cardiac morbidity and mortality after breast cancer treatment is still relevant $[6,7]$. Therefore, dose-volume reduction of irradiation to the heart has become a common treatment goal in order to avoid increased risk of cardiovascular morbidity and

Received 23 August 2013, Revised 7 October 2013, Accepted 15 October 2013.

Correspondence: Ki Chang Keum, MD, PhD, Department of Radiation Oncology, Yonsei Cancer Center, Yonsei University College of Medicine, 50 Yonsei-ro, Seodaemun-gu, Seoul 120-749, Korea. Tel: +82-2-2228-8112, Fax: +82-2-2227-7823, E-mail:kckeum@yuhs.ac

(c) This is an Open Access article distributed under the terms of the Creative Commons Attribution Non-Commercial License (http://creativecommons.org/ licenses/by-nc/3.0/) which permits unrestricted non-commercial use, distribution, and reproduction in any medium, provided the original work is properly cited.

www.e-roj.org 
mortality.

A promising method for minimizing the volume of the heart included in the treatment field involves increasing the distance between the target and heart through deep inspiration. In the deep inspiration breath hold (DIBH) technique, the goal is to achieve the maximum separation between the target area and heart, with irradiation therefore taking place only at or near maximum inspiration. This allows high doses of radiation to be delivered to the chest wall and to the breast tissue while reducing the high dose area of the heart. The DIBH technique has been recommended to reduce radiation to the heart by displacing the breast and chest wall away from the heart during treatment [8-11]. Different methods, such as the use of real-time position management systems [12], active breathing control [13], and magnetic sensors [14] have been used to monitor or control respiratory motion. However, these methods have not been routinely implemented by many breast cancer treatment centers. The failure to adopt DIBH into routine practice is due to concern about increases in the required resources and workloads.

We explored whether the DIBH technique using Abches during left-sided breast irradiation was effective for minimizing the amount of radiation to the heart and lung compared to free breathing (FB). This is the first study to evaluate the feasibility of DIBH using the commercially available Abches system and to incorporate this method into routine clinical practice. We also performed dosimetric evaluations to assess the extent of dose reduction to the heart, left anterior descending coronary artery (LAD), and ipsilateral lung.

\section{Materials and Methods}

\section{Patient selection}

Beginning in February 2012, we implemented the DIBH technique using Abches for selected patients undergoing adjuvant radiotherapy after breast-conserving surgery for left-sided invasive or in situ breast carcinoma at our institution. Patient selection criteria for DIBH irradiation were early stage ( $\leq \mathrm{T} 2$ and $\leq \mathrm{N} 1$ a stage) breast cancer for which whole breast irradiation (without regional nodal irradiation) was indicated. Between February and July 2012, the feasibility of 30-second DIBH was evaluated among 26 selected patients by a dedicated technician. Only 1 patient was excluded from use of the DIBH technique due to frequent cough. The remaining 25 patients underwent computed tomography (CT) simulation scans (SOMATOM sensation; Siemens, Erlangen, Germany) with both
$\mathrm{FB}$ and DIBH without intravenous contrast. These scans were retrospectively replanned using standardized criteria for the purpose of this study. The median age of these patients was 29 years (range, 23 to 76 years).

\section{Abches system}

Abches is a respiration-monitoring device that was developed by Onishi et al. at the University of Yamanashi, Japan [15] (Fig 1). The main unit of the Abches system is composed primarily of plastic, with the use of metal kept to an absolute minimum. Fulcrum $A$ and $B$ are placed on the abdomen and breast of the patient, respectively. During respiration, pointer $C$ of the device moves along with the fulcrum. This device therefore serves as an external device to monitor respiration. The Abches system is not used for gating irradiation but for facilitating precise irradiation of a target by assuring a stable breath hold.

\section{Respiratory control using the Abches system}

All patients were advised that the treatment was to be administered during respiratory standstill and were informed about the importance of reproducing the tumor position at the outpatient clinic by the treating physician. Next, each patient was trained how to hold her breath in the supine position with the left arm above the head in a customized arm support using a breast board to ensure that the sternum was in a horizontal position. Fig. 2 shows the procedure implemented for respiratory control using the Abches system. For all patients, the positions of fulcrum $A$ and $B$ were correctly reproduced every time using anatomical landmarks on the patient as

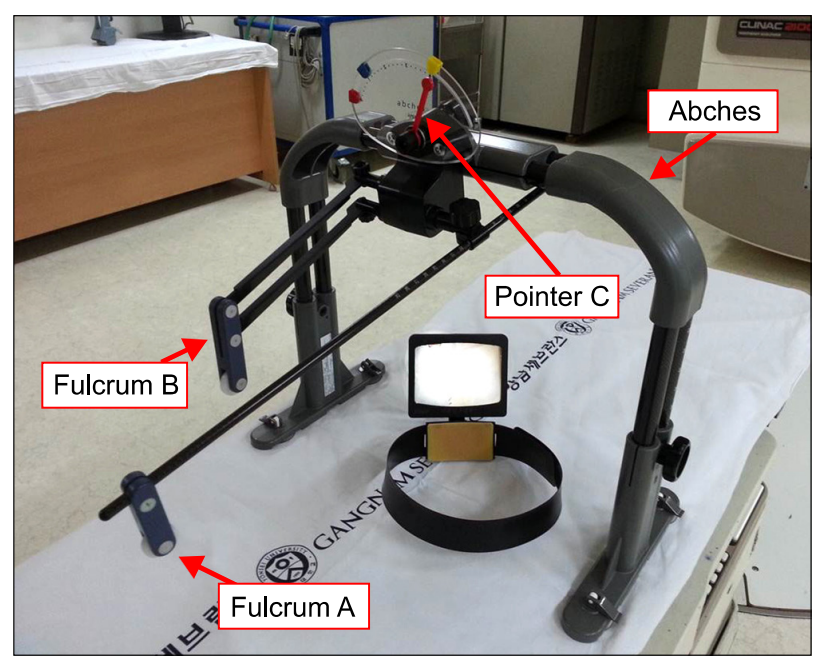

Fig. 1. Abches system. 
indices, the contralateral breast and just below the xiphoid process, respectively. Patients were instructed to hold their breath at the end of inspiration and asked to practice breath holding by repeating the instructions "breathe in, out, and stop" with a mirror in front of the forehead (Fig. 2A). During each respiration session, the position of pointer $C$ shifted with the fulcrum and stopped at some other fixed point. The red and blue marks indicated the ends of expiration and inspiration during regulatory respiration for each patient. The yellow mark indicated the deep inspiration breath hold, which is different for each patient. It was important that each patient maintained her breath hold well at the yellow mark (Fig. 2BD). The monitor was continuously observed during actual CT scanning and irradiation in the operating room. If the position pointer $C$ varied considerably or if the patient could hold her breath for only a few seconds even after repeated training, the use of the Abches system was discontinued for that patient.

\section{Delineation of target volumes and organs at risk}

For consistency, the same physician performed all of the delineation procedures according to the Radiation Therapy Oncology Group guidelines using MIMvista ver. 5.4 (MIM Software, Cleveland, OH, USA), and the same CT threshold values, window levels and widths were used for all scans of all patients. The breast clinical target volume (CTV) included visible glandular breast tissue and the surgical cavity visualized on the CT scan, excluding $5 \mathrm{~mm}$ from the skin; contouring was assisted by a lead wire placed along the palpated breast tissue prior to the CT scan. Medially and laterally, the breast planning target volume (PTV) was defined by the angle of the tangential beams and extended from the midline to the midaxilla. Superiorly and inferiorly, the breast PTV was extended to include visible glandular breast tissue with a margin of $1.5 \mathrm{~cm}$. If the boundary of the breast tissue could not be determined, the superior border was placed at the level of the suprasternal notch. The breast PTV was not adjusted to minimize the volume of the heart included within the tangential fields. The heart contour included the ventricles and atria, including the root of the aorta, the pulmonary artery and veins, and venae cavae. Since cardiac vessels run in the fatty tissue within the pericardium, they were included in the contours. The LAD was delineated from the root of the aorta to the apex of the heart with a standardized 5-mm diameter to account for uncertainties associated with cardiac motion and respiration. Intravenous contrast was not used for the CT scans. However, in most patients, the LAD could be visualized directly, and its position could also be identified according to its location overlying the interventricular septum [16]. The ipsilateral lung was delineated using the CT lung window.

MIMvista ver. 5.4 (MIM Software) is an intensity-based
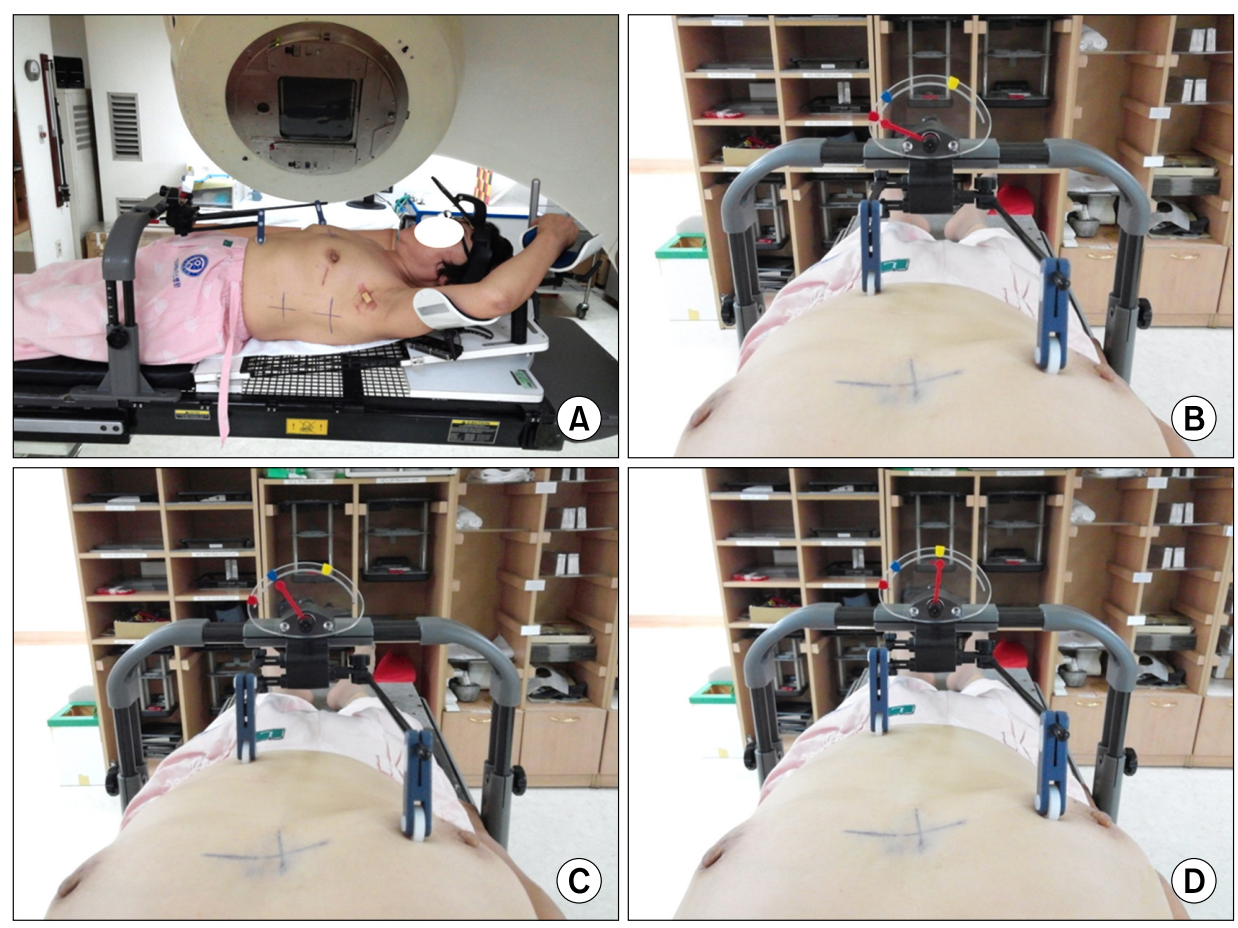

Fig. 2. Procedure for respiratory control using the Abches system. (A) Patient position using Abches. (B) The red mark indicates the end of expiration during regulatory respiration. (C) The blue mark indicates the end of inspiration during regulatory respiration. (D) The yellow mark indicates the deep inspiration breath hold. 
free-form deformable registration algorithm with essentially limitless degrees of freedom designed to expedite the delineation of target volumes and organs at risk. Rigid registration allows linear or uniform transformation of all voxels in the image set within six degrees of freedom (translations in three orthogonal directions and yaw, pitch, and roll rotations). It is of limited utility in the DIBH setting due to significant changes in anatomy. On the other hand, deformable registration algorithms compute the deformation maps of vector fields that can connect the voxels in FB CT images to DIBH CT images, a process that accounts for nonlinear and nonuniform relationships between the image sets. Thus, MIMvista is a useful tool for target volume delineation in breast cancer patients undergoing radiotherapy using the DIBH technique. Deformable registrations of images at our institution were performed with MIMvista. This algorithm was evaluated for clinical use by Piper [17].

\section{Treatment planning and dosimetric evaluation}

For each patient, both FB and DIBH CT scans were planned by a single dosimetrist to ensure plan uniformity. For all CT scans, computerized treatment planning was performed using Pinnacle ver. 9.4 (Philips, Andover, MA, USA). Two opposing 6- or 10-MV tangential conformal fields with multileaf collimators were used. The prescription doses of all plans were $50.4 \mathrm{~Gy}$ in $1.8 \mathrm{~Gy}$ per fraction for the whole breast.

Comparisons of the following dosimetric values for FB and DIBH plans were performed using MIMvista: mean heart dose, the volume of the heart receiving $20 \mathrm{~Gy}$ and $30 \mathrm{~Gy}$ (heart V20 and V30), mean LAD dose, the maximal dose to $0.2 \mathrm{~cm}^{3}$ of the $L A D$, the mean left lung dose and the volume of the left lung receiving $20 \mathrm{~Gy}$ (left lung V20). Because the LAD is a serial structure, the maximal dose to $0.2 \mathrm{~cm}^{3}$ of the $L A D$, which is approximately $1 \mathrm{~cm}$ in length using the standardized $5-\mathrm{mm}$ diameter and may be more biologically relevant than the point maximal dose, was evaluated. Paired t-tests were performed using SPSS ver. 20.0 (SPSS Inc., Chicago, IL, USA) to compare dosimetric parameters between treatment techniques. A twotailed $p<0.05$ was considered statistically significant.

\section{Results}

\section{Patient compliance}

After excluding only 1 patient from the DIBH technique because of frequent cough, the remaining 25 patients were successfully treated with the DIBH technique using Abches. The procedure was easily comprehended by patients. As noted earlier, because the patients were able to watch their own breathing by looking directly into a mirror, it was simple to educate the patients and the patients learned quickly. The mean monitor unit (MU) for two opposing 6- or 10-MV tangential plan was about $213 \mathrm{MU} / \mathrm{min}$ (range, 196 to 265 $\mathrm{MU} / \mathrm{min}$ ) and the beam-on time for a dose rate of $400 \mathrm{MU} / \mathrm{min}$ was about 15 seconds. Thus, the breath hold was well accepted with a duration of about 15 seconds. All 25 patients were able to produce at least three consecutive breath holds without problems.

Table 1. Dosimetric comparison between FB and DIBH plans for all 25 patients

\begin{tabular}{lccc}
\hline \multicolumn{1}{c}{ Variable } & FB plan & DIBH plan & p-value \\
\hline Heart & & & \\
Mean dose (Gy) & $4.53(1.15-8.73)$ & $2.52(0.70-6.03)$ & $<0.001$ \\
V30 (\%) & $6.31(0.25-13.84)$ & $2.80(0.00-10.60)$ & $<0.001$ \\
V30 absolute volume $\left(\mathrm{cm}^{3}\right)$ & $45.13(1.21-97.32)$ & $16.48(0.00-64.45)$ & $<0.001$ \\
V20 (\%) & $7.64(0.64-15.94)$ & $3.16(0.00-10.94)$ & $<0.001$ \\
V20 absolute volume $\left(\mathrm{cm}^{3}\right)$ & $54.55(3.09-110.45)$ & $21.35(0.00-74.14)$ & $<0.001$ \\
LAD & & & \\
Mean dose (Gy) & $26.26(3.82-48.97)$ & $16.01(1.84-31.22)$ & $<0.001$ \\
Dmax 0.2 $\mathrm{cm}^{3}(\mathrm{~Gy})$ & $47.27(17.50-54.05)$ & & 0.017 \\
Lung & & & \\
Mean dose (Gy) & $8.04(5.40-12.19)$ & $14.63(5.03(10.30-10.43)$ & 0.073 \\
V20 (\%) & $15.72(10.11-23.65)$ & $182.76(76.43-342.84)$ & 0.060 \\
V20 absolute volume $\left(\mathrm{cm}^{3}\right)$ & $279.73(163.06-635.73)$ & & 0.059
\end{tabular}

Values are presented as mean (range).

$F B$, free breathing; $D I B H$, deep inspiration breath hold; $L A D$, left anterior descending coronary artery.

a) Paired t-test. 


\section{Dosimetric comparison between FB and DIBH plans}

As shown in Table 1, significant differences were found between the DIBH and FB plans for the mean heart dose (2.52 vs. 4.53 Gy), heart V30 (16.48 vs. $\left.45.13 \mathrm{~cm}^{3}\right)$, V20 (21.35 vs. $\left.54.55 \mathrm{~cm}^{3}\right)$, mean LAD dose (16.01 vs. $26.26 \mathrm{~Gy}$, all $p<0.001$ ), and maximal dose to $0.2 \mathrm{~cm}^{3}$ of the LAD (41.65 vs. $47.27 \mathrm{~Gy}, \mathrm{p}=0.017$ ). In Fig. 3, beams-eye views are shown for the medial tangential fields of left-sided breast cancer patients for both FB and DIBH. Cardiac volume within the radiation field was minimized as much as possible, primarily by conforming the posterior borders of the tangential fields to the CTV using multileaf collimators. The use of DIBH allowed the left-sided breast and heart to be separated during radiation treatment, thus excluding substantial heart volumes from the high dose area. This is demonstrated by the calculated dose volume histogram (Fig. 4). There were significantly lower doses delivered to the heart and LAD with the DIBH plan compared to the FB plan. Although lung inflation during DIBH increased the absolute lung volume within the field on beams-eye view (Fig. 3), the mean left lung dose (7.53 vs. $8.03 \mathrm{~Gy}, \mathrm{p}=0.073$ ) and lung V20 (14.63\% vs. $15.72 \%, p=0.060$ ) were reduced with DIBH compared to the FB plan (Table 1), although the difference was not significant.

\section{Workload requirements and patient and staff acceptance}

The implementation of the DIBH technique required minimal additional resources and only a moderate increase in workload.
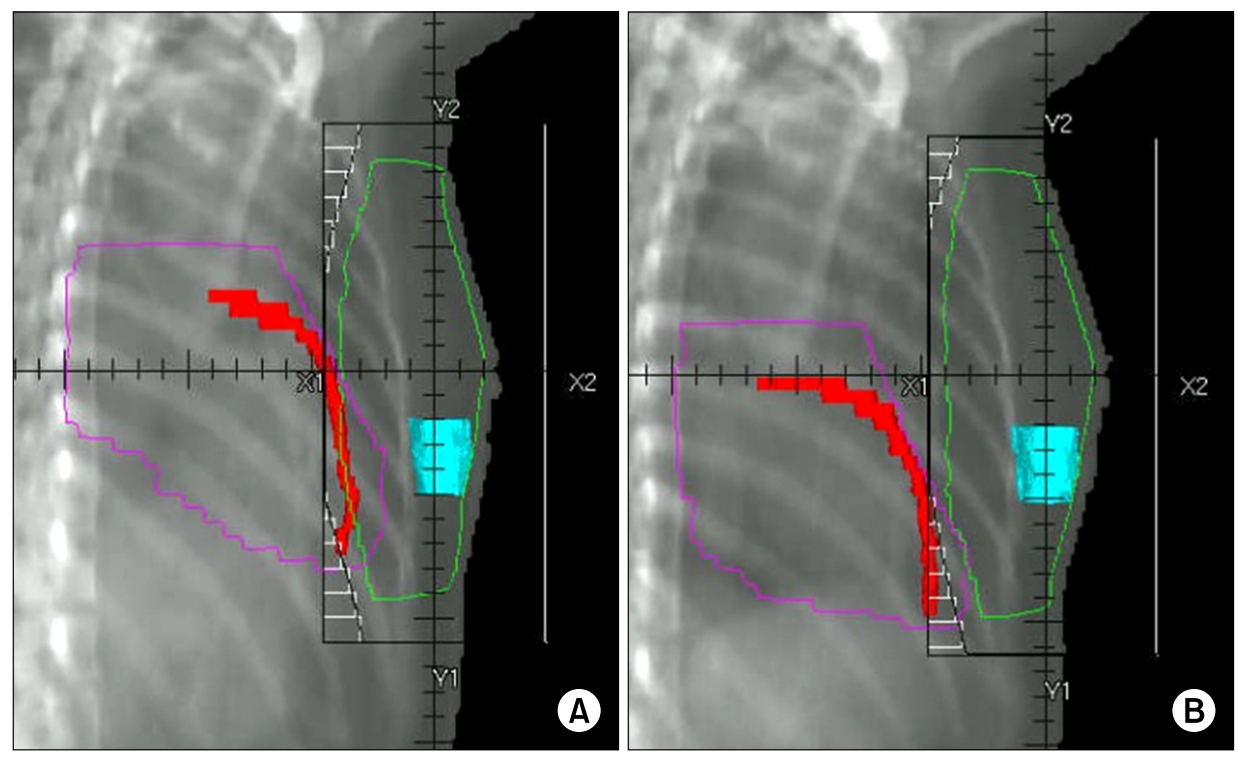

Fig. 3. Beams-eye views of the medial tangential fields for free breathing $(A)$ and deep inspiration breath hold (B) plans. Heart outline in pink, left anterior descending coronary artery in red, and surgical cavity in sky blue.

A

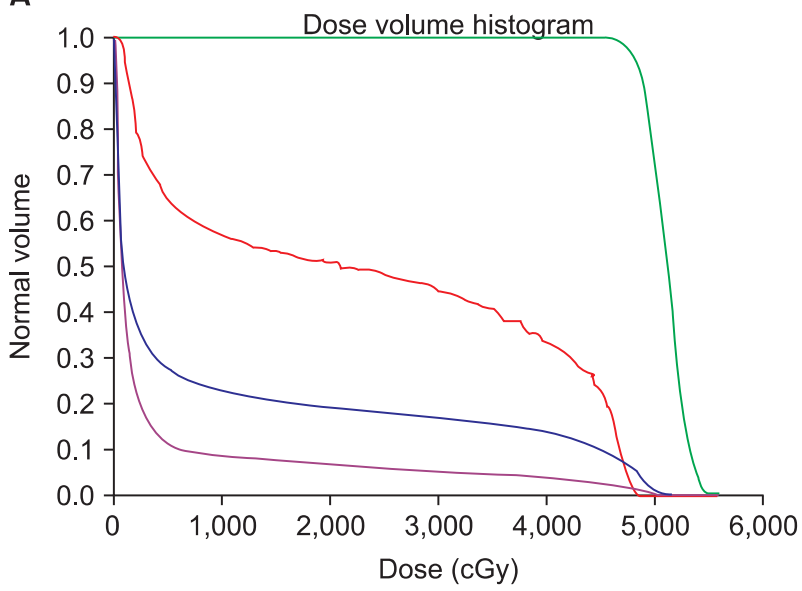

B

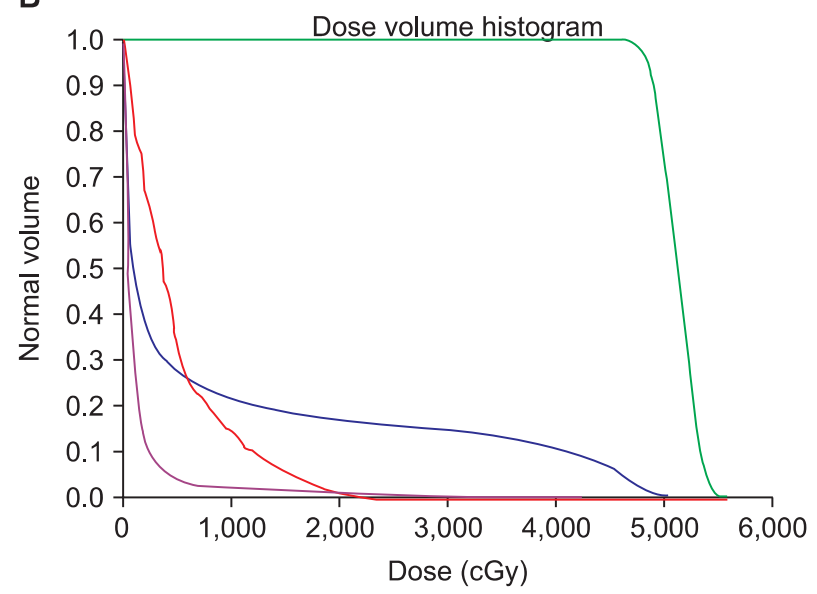

Fig. 4. Dose volume histograms comparing free breathing (A) and deep inspiration breath hold (B) plans for the same patient. Whole breast line in green, left anterior descending coronary artery in red, heart in pink, and ipsilateral lung in blue. 
We assessed the overall treatment time for each patient. The mean durations of FB and DIBH were about 5 minutes 10 seconds and 10 minutes 30 seconds, respectively. All planning staff became familiar with the DIBH planning and Abches education processes. Staff responsible for operating breast cancer treatment machines became proficient with patient setup and treatment delivery using Abches in about 6 weeks.

\section{Discussion and Conclusion}

Delivering treatment to the left breast using the $\mathrm{DIBH}$ technique has been shown to reduce both the irradiated heart volume and dose to the heart. Early studies indicated that $\mathrm{DIBH}$ could reduce the volume of the heart within tangential fields. Remouchamps et al. [8] reported a mean absolute reduction in heart $\mathrm{V} 30$ of $3.6 \%$ in a preliminary study including five patients. Korreman et al. [18] reported a reduction in the median heart volume receiving more than $50 \%$ of the prescription dose from $19.2 \%$ to $1.9 \%$ and a reduction in the median volume of the $L A D$ receiving the same dose from $88.9 \%$ to $3.6 \%$ in a sample of nine patients. In a subsequent publication, the same group used the relative seriality model to calculate the expected reduction in cardiac mortality from the use of DIBH in 16 cases. The cardiac mortality probabilities for $\mathrm{FB}$ and $\mathrm{DIBH}$ were $4.8 \%$ and $0.1 \%$ [12].

More recent reports support these findings. At Princess Margaret Hospital, Toronto, Canada, 20 patients were planned for both FB and DIBH treatments. Statistically significant reductions in the volume of the heart receiving more than $50 \%$ of the prescription dose, mean heart dose, mean LAD dose, and the maximum dose to $0.2 \mathrm{~cm}^{3}$ of the LAD were noted after applying the DIBH technique [11]. In a similar study of 19 unselected patients from the Netherlands Cancer Institute the maximum and mean doses and the V30 for the heart, the left ventricle and the LAD were all significantly reduced by DIBH [10]. Several reports have documented the tolerability and interfraction reproducibility of the DIBH technique $[13,19,20]$.

Our data are consistent with those of other published reports demonstrating significant reductions in the doses to the heart and LAD with DIBH technique using Abches. However, several factors may limit comparisons between studies. The reported heart doses may be influenced by variability in the method used to contour the heart between studies. We employed noncontrast CT scans to outline the atria and ventricles. Previous studies excluded the epicardial fat and pericardium, the roots of the great vessels and/or the venae cavae, and were performed with or without the use of intravenous contrast. Contouring the coronary arteries is particularly difficult, as they are often poorly visualized due to non-contrast CT scans and often seem noncontiguous throughout the data set due to cardiac and respiratory motion. To account for these uncertainties, we used a standardized contouring protocol [16]. We attempted to maintain consistency of volume measurements by employing the same radiation oncologist to perform all contouring. Even so, some minor variations in all contours may have occurred due to variation in the anatomy of the heart and lung between FB and DIBH scans.

Although the absolute left lung volume appeared to have increased with DIBH, proportionately less of the left lung was irradiated. While lung volume may increase with deep inspiration, the lung density may decrease, resulting in irradiation of a reduced fraction of normal lung mass [21]. In our study, the mean left lung dose (7.53 vs. $8.03 \mathrm{~Gy}, \mathrm{p}=$ $0.073)$ and percentage of the left lung (14.63\% vs. $15.7 \%$, $p=0.060$ ) receiving 20 Gy were both smaller with $\mathrm{DIBH}$ (borderline significance). Therefore, the risk of radiation-induced pneumonitis is unlikely to increase using the DIBH technique.

Implementing the DIBH technique using Abches for all patients receiving left-sided breast radiotherapy was neither desirable nor feasible, because the benefits were questionable for most patients with minimal or no heart included within the radiation field. Therefore, it is important to establish criteria to select patients with unfavorable cardiac anatomy, who would benefit most from using Abches. Our results demonstrate that $\mathrm{DIBH}$ technique using Abches resulted in statistically significant reductions in the mean heart dose, heart V2O, heart V30, mean LAD dose, and maximal dose to $0.2 \mathrm{~cm}^{3}$ of the LAD. These parameters may be of assistance in identifying patients with unfavorable cardiac anatomy who would most benefit from reductions in cardiac dose during radiotherapy to the left breast. Further research is needed to determine which patients are likely to derive the most benefit from this technique before DIBH CT scans are performed.

It has been demonstrated in several studies that breathing adaptation techniques can be used to reduce the irradiated heart and lung volumes, primarily by utilizing lung inflation which dilutes the amount of lung tissue in the radiation fields and spatially separates the heart from target. An active breathing control device has been used to freeze the lung volume at $75 \%$ of maximum inspiration capacity, providing significant lung and heart dose reductions [22]. The device consists of a digital spirometer to record the patients' real- 
time breathing trace and a balloon valve that is triggered to automatically inflate when the patient inspire to their preset DIBH level. This allows for a predictable, consistent level of chest wall expansion with each DIBH. Another one of breathing adaptation techniques is the Real-time Position Management (RPM) system (Varian Medical Systems, Palo Alto, CA, USA). The device permits breathing-synchronized CT scanning and radiotherapy during certain time intervals on the linear accelerator [18]. The position and chest wall excursion are assessed individually for each breath-hold mode, and the patient is then instructed to repeat each mode to improve reproducibility. We think that Abches would probably be less precise than other breathing adaptation techniques in terms of consistent level of chest wall expansion and reproducibility of breath-hold level.

However, because the patient can monitor her own breathing and breath holds with durations of about 15 seconds were well accepted, the breathing procedure was easily comprehensible to the patients and there was good compliance using Abches. In addition, the main unit of the Abches system is composed primarily of plastic and is therefore quite light. Thus, it is easy to perform patient setup in the radiation treatment room without affecting dose calculation using the radiation treatment planning system (Pinnacle ver. 9.4, Philips). All planning staff easily became familiar with the DIBH planning and Abches education processes. The staff who operated breast cancer treatment machines became proficient with patient setup and treatment delivery using Abches after about 6 weeks. The additional workload per patient treated with DIBH using Abches was modest, and the use of MIMvista dramatically reduced the breast radiation treatment planning time, allowing rapid delineation and dose-volume assessments of organs at risk in real-time using a FB plan. This procedure can be implemented for routine use in busy clinics.

In conclusions, we report that the application of the DIBH technique using Abches in breathing adapted radiotherapy for left-sided breast cancer is easily feasible in daily practice and significantly reduces the doses to the heart and LAD, therefore potentially reducing the cardiac risk. Based on the results of this study, at our institution we now treat left-sided breast cancer patients with Abches.

\section{Conflict of Interest}

No potential conflict of interest relevant to this article was reported.

\section{Acknowledgments}

The present research has been supported by the Korea Breast Cancer Foundation. The authors thank the radiologic technicians for their valuable help with patient setup.

\section{References}

1. Hooning MJ, Botma A, Aleman BM, et al. Long-term risk of cardiovascular disease in 10-year survivors of breast cancer. J Natl Cancer Inst 2007;99:365-75.

2. Offersen B, Hojris I, Overgaard M. Radiation-induced heart morbidity after adjuvant radiotherapy of early breast cancer: Is it still an issue? Radiother Oncol 2011;100:157-9.

3. Early Breast Cancer Trialists' Collaborative Group. Favourable and unfavourable effects on long-term survival of radiotherapy for early breast cancer: an overview of the randomised trials. Lancet 2000;355:1757-70.

4. Correa CR, Litt HI, Hwang WT, Ferrari VA, Solin $\sqcup$, Harris EE. Coronary artery findings after left-sided compared with rightsided radiation treatment for early-stage breast cancer. J Clin Oncol 2007;25:3031-7.

5. Darby SC, Ewertz M, McGale P, et al. Risk of ischemic heart disease in women after radiotherapy for breast cancer. $\mathrm{N}$ Engl J Med 2013;368:987-98.

6. Demirci S, Nam J, Hubbs JL, Nguyen T, Marks LB. Radiationinduced cardiac toxicity after therapy for breast cancer: interaction between treatment era and follow-up duration. Int J Radiat Oncol Biol Phys 2009;73:980-7.

7. Darby SC, McGale P, Taylor CW, Peto R. Long-term mortality from heart disease and lung cancer after radiotherapy for early breast cancer: prospective cohort study of about 300,000 women in US SEER cancer registries. Lancet Oncol 2005;6:55765.

8. Remouchamps VM, Letts N, Vicini FA, et al. Initial clinical experience with moderate deep-inspiration breath hold using an active breathing control device in the treatment of patients with left-sided breast cancer using external beam radiation therapy. Int J Radiat Oncol Biol Phys 2003;56:704-15.

9. Nissen HD, Appelt AL. Improved heart, lung and target dose with deep inspiration breath hold in a large clinical series of breast cancer patients. Radiother Oncol 2013;106:28-32.

10. Borst GR, Sonke JJ, den Hollander S, et al. Clinical results of image-guided deep inspiration breath hold breast irradiation. Int J Radiat Oncol Biol Phys 2010;78:1345-51.

11. Wang W, Purdie TG, Rahman M, Marshall A, Liu FF, Fyles A. Rapid automated treatment planning process to select breast cancer patients for active breathing control to achieve cardiac dose reduction. Int J Radiat Oncol Biol Phys 2012;82:386-93. 
12. Korreman SS, Pedersen AN, Aarup LR, Nottrup TJ, Specht L, Nystrom $\mathrm{H}$. Reduction of cardiac and pulmonary complication probabilities after breathing adapted radiotherapy for breast cancer. Int J Radiat Oncol Biol Phys 2006;65:1375-80.

13. Moran JM, Balter JM, Ben-David MA, Marsh RB, Van Herk M, Pierce $\sqcup$. Short-term displacement and reproducibility of the breast and nodal targets under active breathing control. Int J Radiat Oncol Biol Phys 2007;68:541-6.

14. Remouchamps VM, Huyskens DP, Mertens I, et al. The use of magnetic sensors to monitor moderate deep inspiration breath hold during breast irradiation with dynamic MLC compensators. Radiother Oncol 2007;82:341-8.

15. Tarohda $\mathrm{TI}$, Ishiguro $M$, Hasegawa $K_{1}$ et al. The management of tumor motions in the stereotactic irradiation to lung cancer under the use of Abches to control active breathing. Med Phys 2011;38:4141-6.

16. Feng $M$, Moran JM, Koelling $T$, et al. Development and validation of a heart atlas to study cardiac exposure to radiation following treatment for breast cancer. Int J Radiat Oncol Biol Phys 2011; 79:10-8.

17. Piper J. Evaluation of an intensity-based free-form deformable registration algorithm. Med Phys 2007;34:2353-4.
18. Korreman SS, Pedersen AN, Nottrup TJ, Specht L, Nystrom H. Breathing adapted radiotherapy for breast cancer: comparison of free breathing gating with the breath-hold technique. Radiother Oncol 2005;76:311-8.

19. Remouchamps VM, Letts $N$, Yan D, et al. Three-dimensional evaluation of intra- and interfraction immobilization of lung and chest wall using active breathing control: a reproducibility study with breast cancer patients. Int J Radiat Oncol Biol Phys 2003;57:968-78.

20. Jagsi R, Moran JM, Kessler ML, Marsh RB, Balter JM, Pierce $\sqcup$. Respiratory motion of the heart and positional reproducibility under active breathing control. Int J Radiat Oncol Biol Phys 2007;68:253-8

21. Sixel KE, Aznar MC, Ung YC. Deep inspiration breath hold to reduce irradiated heart volume in breast cancer patients. Int J Radiat Oncol Biol Phys 2001;49:199-204.

22. Remouchamps VM, Vicini FA, Sharpe MB, Kestin LL, Martinez AA, Wong JW. Significant reductions in heart and lung doses using deep inspiration breath hold with active breathing control and intensity-modulated radiation therapy for patients treated with locoregional breast irradiation. Int J Radiat Onco Biol Phys 2003;55:392-406. 